\title{
A P Cygni Profile for the He 10830 A line of CI Cyg in Eclipse
}

\author{
S. Bensammar ${ }^{1}$, M. Friedjung ${ }^{2}$, N. Letourneur ${ }^{1}$, J.P. Maillard ${ }^{2}$ \\ 1 Observatoire de Paris, section de Meudon \\ UA 337 du CNRS, 92195 Meudon Principal Cedex, France \\ 2 Institut d'Astrophysique de Paris, CNRS \\ 98 bis, bvd Arago 75014 Paris, France
}

\begin{abstract}
Infrared spectroscopic observations of CI Cyg in eclipse show a P Cygni profile for He I $10830 \AA$, with a wind velocity of the order of $150 \mathrm{~km} / \mathrm{s}$, not seen in hydrogen Brackett and Paschen emission lines, which are single peaked.
\end{abstract}

\section{OBSERVATIONS}

We report here very early results of high-resolution observations of the eclipsing symbiotic star CI Cyg in the infrared (1 to $2.5 \mu \mathrm{m})$ made in July 1987 , just at the time of an eclipse of the hot component by the cool component ( phase 0 ). The observations were performed with the Cassegrain Fourier Transform Spectrometer of the Canada - France - Hawaii Telescope (Maillard and Michel 1982), through the standard filters $\mathrm{J}(1.25 \mu \mathrm{m}), \mathrm{H}(1.65 \mu \mathrm{m})$ and $\mathrm{K}(2.25 \mu \mathrm{m})$, at a resolution ranging roughly from 20,000 to 40,000 . These observations follow similar observations made during the same cycle at phase 0.5 (Bensammar et al 1987), allowing a comparison of the observed features, and in particular the hydrogen and helium lines, in a different configuration of the binary system. The most striking fact in the phase 0.5 observation was the detection of $\operatorname{Br} \gamma(2.15 \mu \mathrm{m})$ seen in absorption, which was interpreted as the absorption of the infrared thermal radiation of the cool component by an accretion disk surrounding the hot component.

In the new spectra, the most surprising feature is the profile of the He $10830 \AA$ line, which exhibits a very strong P Cygni shape (fig. 1), which was absent at phase 0.5. Such a profile to our knowledge, is reported for the first time for this star. The terminal velocity of the blue absorption is as high as $150 \mathrm{~km} / \mathrm{s}$.

Other prominent lines in these spectra, which all belong to hydrogen, $\mathrm{Br} \gamma, \operatorname{Pa} \beta$ and $\mathrm{Pa} \gamma$ (fig 2,3 and 4), are detected in emission. These lines are not totally eclipsed, which is consistent with previous observations in the the visible of the Balmer lines (Oliversen et al 1982, Micholajewska 1985 ) at phases in and near eclipse, while the Balmer continuum at $3000 \mathrm{~A}$ is totally eclipsed (Stencel et al 1982). The $\mathrm{Br} \gamma$ line has a single peak, while asymmetrical double peak structure, with a deep blueshifted reversal near the time of the total eclipse was observed in $\mathrm{H} \alpha$ (Oliversen et al 1982). No high-resolution profiles of higher Balmer lines are reported to allow a comparison. The strong absorptions seen, one in the red wing of $\mathrm{Pa} \beta$ (fig.3), the other one in the blue wing of $\mathrm{Pa} \gamma$ (fig.4), are not part of the hydrogen line profiles. The profiles presented result from the division of the observed spectrum by that of a reference star, HR 6702, chosen to have a spectral type as close as possible to the type reported for the cool giant of CI 
Cyg. From Kenyon and Fernandez-Castro (1987) the giant is quoted as of type M4.9 \pm 0.7 and class II whereas HR 6702 is identified in catalogues as M5 II. Taking the ratio of the CI Cyg spectrum to the giant star's spectrum should allow one to obtain a pure spectrum of the ionized component, providing the cool component is identical with the reference giant. In addition, the respective Doppler shifts have to be perfectly compensated. A better compensation of Doppler shift should reduce the residual absorptions, on the figures shown. However, comparing the spectra before division shows that differences appear between the two spectra. Some lines are deeper or present only in the CI Cyg spectrum, which makes a perfect compensation impossible. This is particularly true in the Par region. At the present stage of analysis, no conlusion can be drawn as to whether the Paschen lines are symmetrical or not. It can only be stated that they do not exhibit a $P$ Cygni profile and most probably are also single peaked.

\section{DISCUSSION}

The stationary absorption detected at phase 0.5 for $\mathrm{Br} \gamma$ is no longer present at phase 0 , in agreement with our prediction that it should not be seen at phases other than 0.5 , if it is produced by material associated with an accretion disk. The fact that $\mathrm{Pa} \beta$ and $\mathrm{Pa} \gamma$ are strong in these last spectra, unlike the phase 0.5 observation, can be interpreted as due to the absence of the previous absorption which no longer hides the emission.

The clear difference of profile between hydrogen and helium raises the questions of the excitation mechanism of these lines by the radiation of the hot component, the origin of the P Cygni profile seen in the helium line, and of the respective sizes of the $\mathrm{HI}$ and HeI emission regions. A P Cygni profile is traditionally interpreted as the sign of a line formed in an expanding envelope. If the emission lines are formed as a result of the ionization of the cool star's envelope, the ionization front must reach uneclipsed regions of this envelope. The expansion velocity $(\sim 150 \mathrm{~km} / \mathrm{s})$ measured in helium between the observer and the cool star, cannot be attributed to the normal expansion velocity of the cool star's wind, which should be rather of the order of $15 \mathrm{~km} / \mathrm{s}$ (Bernat et al 1979). Another mechanism to accelerate the gas has to be advocated, such as a shock wave. The presence of a P Cygni profile for the He $10830 \AA$ could be connected with the fact that the lower level is metastable, which may permit formation of the absorption component at large distances from the binary.

The difference between the cool component spectrum and a normal $\mathrm{M}$ giant star spectrum, only detectable at high resolution, suggests a different evolutionary status for the giant component of the symbiotic star. A complete study of the stellar absorbers has to be performed in order to deduce relevant abundance ratios like $\mathrm{C} / \mathrm{O},{ }^{12} \mathrm{C} /{ }^{13} \mathrm{C}$ which should lead to decisive clues on this important question.

\section{REFERENCES}

Bensammar, S., Friedjung, M., Letourneur, N., Maillard, J.P., 1987, Astron. Astrop., in press

Bernat, A.P., Hall, D.N.B., Hinkle, K.H., Ridgway, S.T., 1979, Ap. J. (Letters ), 233 , L135.

Kenyon, S.J., Fernandez-Castro, T., 1987, Astron. J., 93, 938.

Maillard, J.P. and Michel, G., 1982, in IAU Colloquium No 92, Instrumentation for Astronomy with large Telescopes, ed. C.M. Humphries, (D. Reidel Pub.), p. 213. 
Mikolejewska, J., Acta Astron. 35, 65.

Oliversen, N.A., Anderson, C.M., Nordsieck, K.H., 1982, in IAU Colloquium No 70 The Nature of Symbiotic Stars, ed By M. Friedjung and R. Viotti (D. Reidel Pub), p.153.

Stencel, E.S., Michalitsianos, A.G., Kafatos, M., Boyarchuk A.A., 1982, Ap. J. Letters, 253, L77.

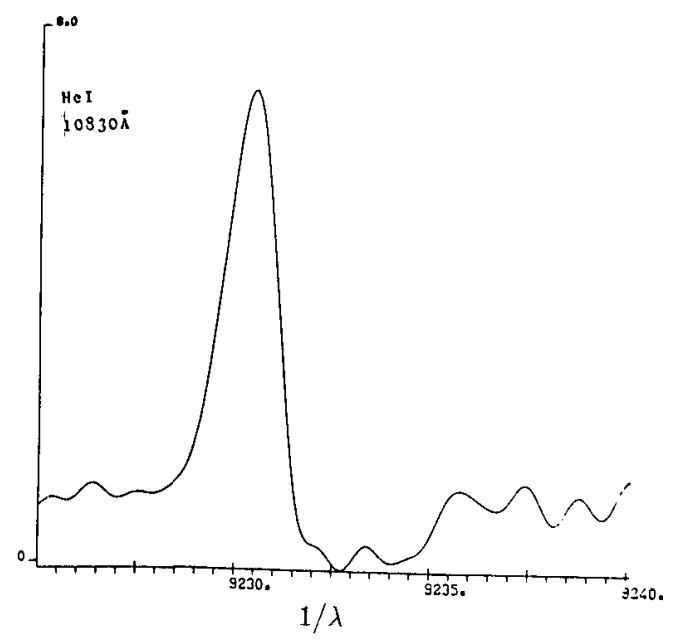

Fig. 1

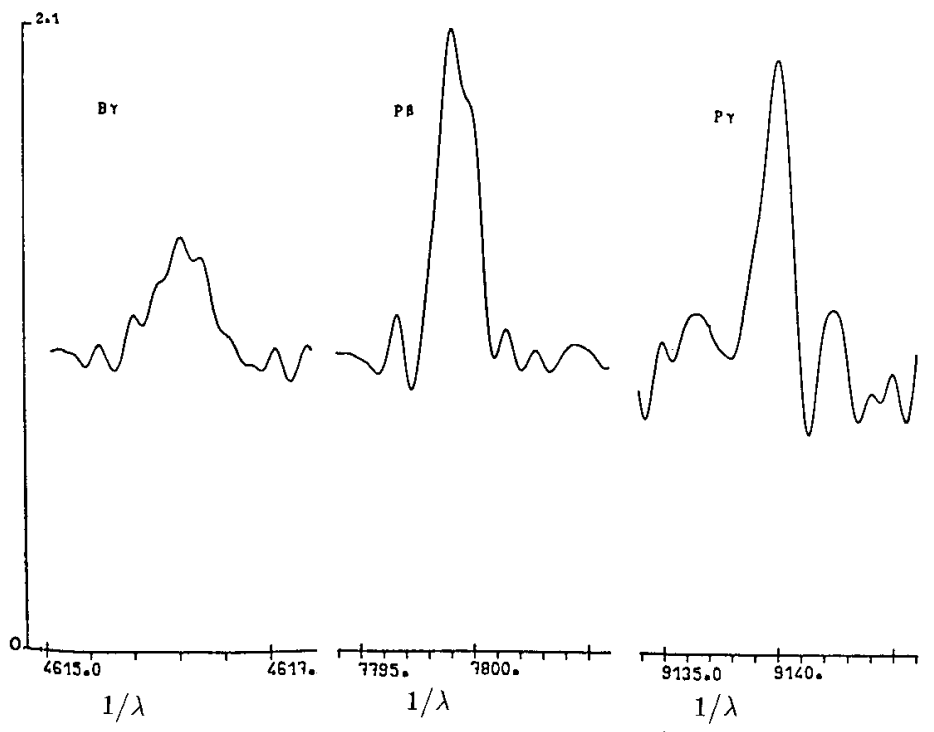

Fig. 2

Fig. 3

Fig. 4 